\title{
Immunohistochemical evidence for proteolipid protein and nestin expression in the late bell stage of developing rodent teeth
}

\author{
T. Struys ${ }^{a, *}$, T. Krage ${ }^{b}$, F. Vandenabeele ${ }^{a}$, W.H.-M. Raab ${ }^{b}$, I. Lambrichts ${ }^{a}$
}

aLimburgs Universitair Centrum, Biomedisch Onderzoeksinstituut and Transnationale

Universiteit Limburg, School for Life Sciences, Diepenbeek, Belgium

${ }^{\mathrm{b}}$ Department of Operative/Preventive Dentistry and Endodontics, Heinrich Heine University,

Duesseldorf, Germany

Accepted 2 November 2004

\author{
KEYWORDS \\ Immunohisto- \\ chemistry; \\ PLP; \\ Nestin; \\ Tooth formation
}

\begin{abstract}
Summary In this study, the expression of proteolipid protein (PLP) and nestin is studied in the late bell stage of developing rodent teeth in neonatal rats. By using immunohistochemistry, it was shown that odontoblasts, ameloblasts and the stratum intermedium are positive for PLP in regions of active matrix deposition. Reactivity for nestin could be detected in the odontoblasts, stratum intermedium and in some of the apical processes of the ameloblasts. The fact that mutations in the PLP gene can cause disturbances in tooth form, number and eruption taken together with the presence of PLP reactivity in odontoblasts and ameloblasts of healthy animals, suggests a crucial role for PLP in developing teeth because of its structural supportive characteristics. These results also imply the possible use of PLP antibody as a new marker for, respectively, dentin and enamel-secreting odontoblasts and ameloblasts. PLP and nestin expression could point to a possible similarity in function between the oligodendrocyte and the odontoblast, both derived from the neural crest. To compare with the situation in human tissue, PLP and nestin expression were preliminarily tested on human dental pulp. The odontoblasts were positive for both PLP and nestin. (C) 2004 Elsevier Ltd. All rights reserved.
\end{abstract}

\section{Introduction}

Proteolipid protein (PLP), encoded by the proteolipid protein gene, is a major structural component of

* Corresponding author. Tel.: +32 478751262; fax: +32 11268599 .

E-mail address: tom.struys@luc.ac.be (T. Struys). the central nervous system (CNS) myelin. ${ }^{1}$ In man, mutations of this gene can cause duchenne muscular dystrophy (DMD), a rapidly progressing degenerative disease that causes severe muscle wasting. ${ }^{2}$ The oral manifestations seen in DMD patients include macroglossia, open bite, expansion of the maxillary and mandibular arches, abnormal function of the facial muscles, delayed tooth emergence and possibly 
agenesis of the second premolar. ${ }^{2}$ To date, no data are available on the expression of PLP in odontoblasts.

Nestin is a protein belonging to a distinct sixth class of intermediate filaments predominantly expressed at early stages of CNS and muscle development. Nestin plays an important role in induction, morphogenesis and differentiation of organs. It is down-regulated in mature tissues and re-expressed in pathological conditions. Previous studies have shown that nestin is expressed in developing and adult human teeth under normal and pathological conditions. ${ }^{3}$ Nestin is accepted to be a specific marker for odontoblasts reflecting its neural-crest derivation. ${ }^{4}$

\section{Materials and methods}

Jaw bones with developing teeth were cut into $2 \mathrm{~mm}$ slices, fixed with formalin 4\% (Unifix ${ }^{\circledR}$, Klinipath) for $1 \mathrm{~h}$, embedded in paraffin and sectioned at $4 \mu \mathrm{m}$. Paraffin sections were mounted on poly-L-lysinecoated glass slides. Sections were deparaffinized and immunostained using an indirect visualization system, based on a peroxidase-labelled polymer (Dako EnVision ${ }^{\mathbb{R}}$ System). All procedures were performed at room temperature. Endogeneous peroxidase was quenched with $0.5 \% \mathrm{H}_{2} \mathrm{O}_{2}$ in methanol. Sections were incubated for $1 \mathrm{~h}$ with either mouse anti-nestin monoclonal antibody (Chemicon) at a dilution of $1 / 2000$ in PBS or mouse anti-PLP monoclonal antibody (Sigma) at a dilution of $1 / 20$ in PBS. Peroxidase activity was revealed with diaminobenzidine (DAB) as a chromogenic substrate. Nuclei were counterstained using Mayers' hematoxylin.
Immunohistochemistry for PLP and nestin was also performed on human dental pulp. Therefore, we used permanent teeth that were extracted for orthodontic or therapeutic reasons. After extraction, the pulp was removed with a forceps and fixed with Unifix ${ }^{\circledR}$, embedded in paraffin and sectioned at $4 \mu \mathrm{m}$. Immunostaining was performed like described above. Antibodies were used at a dilution of $1 / 500$ in PBS for nestin and $1 / 20$ in PBS for PLP. The same detection technique was used.

\section{Results}

\section{PLP}

Strong immunoreactivity was observed in the odontoblast layer, the ameloblast layer and the stratum intermedium (Figs. 1 and 2). In the odontoblasts and ameloblasts, both the cell bodies and the processes exhibited immunoreactivity (Fig. 3). This was only seen in regions of active deposition of dentine and enamel. The cell-rich zone underlying the odontoblasts also stained positive in these regions. At the cervical loop, and in absence of dentine and enamel (right side of dashed line), the odontoblasts and preameloblasts did not present PLP immunolabelling (Fig. 4). Further, immunoreactivity was seen in the external enamel epithelium but not in the stellate reticulum (Fig. 1).

\section{Nestin}

Strong immunoreactivity was seen in both the odontoblast cell bodies and processes (Fig. 5). Immunoreactivity was observed up to the dentin-enamel

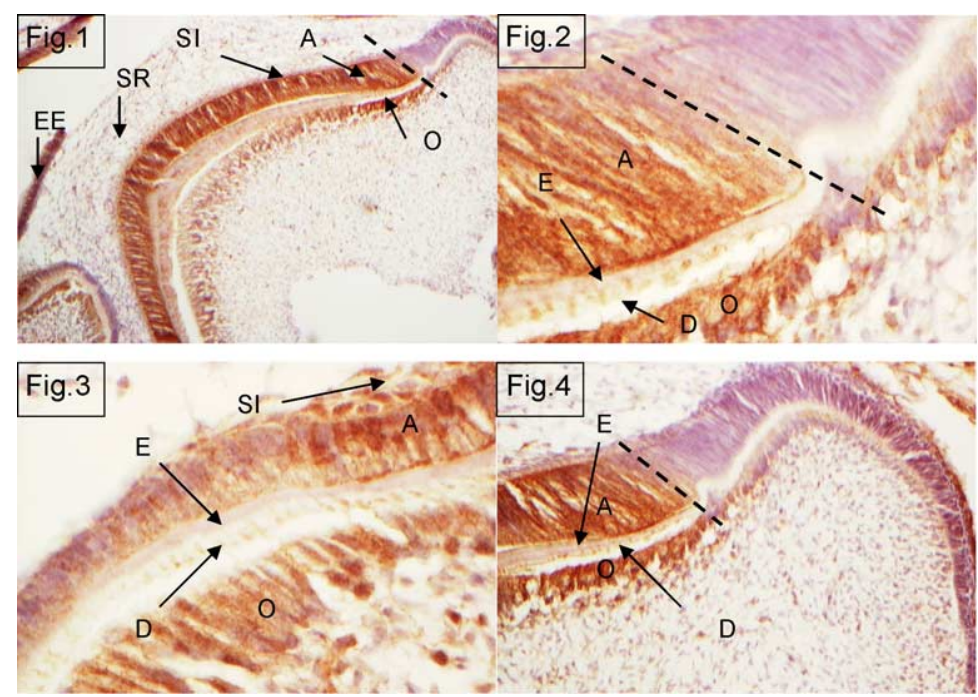

Figures 1-4 A, ameloblasts; E, enamel; O, odontoblasts; D, dentin; SI, stratum intermedium; SR, stellate reticulum; $\mathrm{EE}$, external enamel epithelium. 


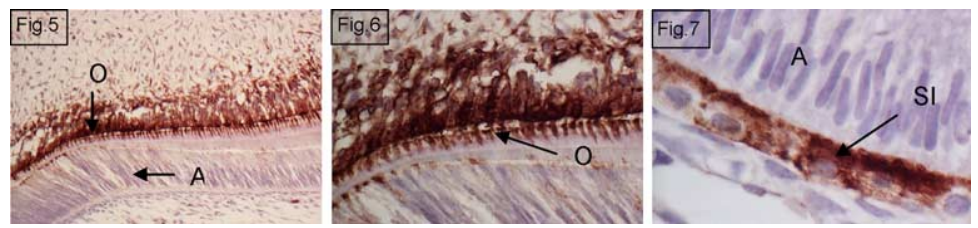

Figures 5-7 A, ameloblasts; O, odontoblasts; OP, odontoblasts processes; SI, stratum intermedium.

junction from the cervical loop to the crown region. Strong nestin immunoreactivity was also observed in the cell-rich zone at the periphery of the pulp (Fig. 6) and in the stratum intermedium (Fig. 7). Some of the apical processes (Tomes' processes) of the ameloblasts were nestin-positive. Immunostaining of Tomes' processes was most pronounced at the cervical loop region and decreased toward the cusp area.

Immunohistochemistry for PLP and nestin on human dental pulp was also performed. Positive staining for PLP and nestin in the odontoblasts was observed (Figs. 8 and 9).

\section{Discussion}

PLP and nestin expression is present in odontoblasts, ameloblasts and stratum intermedium in developing teeth of neonatal rat. We exclude a possible crossreaction between PLP and amelogenin [genes of both proteins are present on the X-chromosome separated by only one gene ${ }^{5}$ due to the specificity of the staining in the regions of active and no deposition of matrix. PLP functions as a mechanic anchor between two cell membranes and in this way PLP is a very important structural protein in the formation of myelin. PLP, present in odontoblasts and ameloblasts, could likely enhance the adherence of both cell layers when they deposite their secretions: dentin and enamel, respectively. In a study concerning dental characteristics of patients with DMD, disturbances in tooth form, number and eruption of the second premolars were observed in $39 \%$ of patients. ${ }^{6}$ Disturbances in premolar tooth

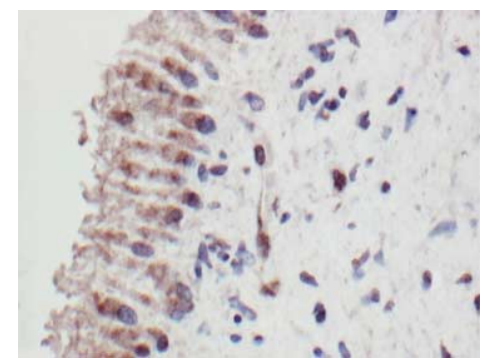

Figure 8 Immunoreactivity for PLP in human dental pulp. Note that the odontoblast layer is positive.

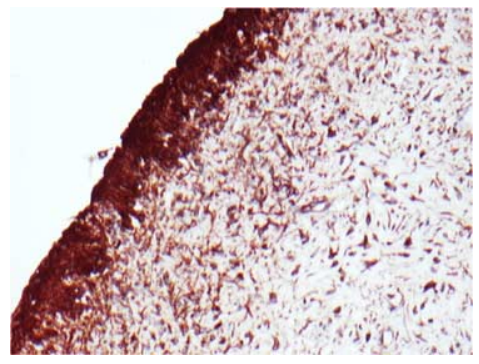

Figure 9 Immunoreactivity for nestin in human dental pulp. The odontoblast layer and the cell-rich zone of the pulp are positive.

eruption and tooth form have been described in association with second premolar agenesis. ${ }^{7,8}$ Agenesis is said to be genetically determined, and may be present as an isolated trait or be associated with a more generalized disturbance. ${ }^{9}$ Defects in tooth eruption have also been linked to genetic conditions affecting tooth development. ${ }^{10}$ It remains to be determined whether the disturbances in tooth eruption and form are variations in expression of the same genetic factor that results in agenesis. One of the key mutations found in the Duchenne syndrome is a duplication of the PLP gene resulting in a failed transcription. ${ }^{2}$ This suggests a crucial role for PLP in the developing tooth. In some other neurodegenerative diseases such as Pelizaeus-Merzbacher disease, where a duplication of the PLP gene is one of the possible causing mutations, ${ }^{11}$ it then might be possible to detect malformation of the dentin and enamel due to the loss of PLP. The immunoreactivity for PLP in mature pulp suggests that the adherence, offered by PLP, might be necessary during secondary dentinogenesis. The specificity of the staining in regions of active deposition supports the hypothesis that PLP could be used as a new marker for active secreting odontoblasts and ameloblasts.

PLP and nestin expression taken together could point to a possible similarity in function between the oligodendrocyte and the odontoblast, both derived from the neural crest. Like the oligodendrocyte, the function of the odontoblast might be supporting the sensory nerves entering the dentinal tubules. Further investigation is necessary to confirm these hypotheses. 


\section{Acknowledgments}

We would like to acknowledge Marc Jans, MarieJosée Sleypen and Marjan Moreels for their technical assistance and advice in performing the immunohistochemistry. We also would like to thank Dr. C. Politis for kindly providing the human teeth.

\section{Reference}

1. Yool DA, Edgar JM, Montague P, Malcolm S. The proteolipid protein gene and myelin disorders in man and animal models. Hum Mol Genet 2000;9(6):987-92.

2. Saito-Ohara F, Fukuda $Y$, Ito $M$, et al. The Xq22 inversion breakpoint interrupted a novel Ras-like GTPase gene in a patient with Duchenne muscular dystrophy and profound mental retardation. Am J Hum Genet 2002;71(3):637-45.

3. About I, Laurent-Maquin D, Lendahl U, Mitsiadis TA. Nestin expression in embryonic and adult human teeth under normal and pathological conditions. Am J Pathol 2000;157(1):287-95.

4. Terling C, Rass A, Mitsiadis TA, Fried K, Lendahl U, Wroblewski $J$. Expression of the intermediate filament nestin during rodent tooth development. Int J Dev Biol 1995;39(6): 947-56.

5. Blair HJ, Reed V, Laval SH, Boyd Y. The locus for pyruvate dehydrogenase E1 alpha subunit (Pdha1) lies between Plp and Amg on the mouse X chromosome. Mamm Genome 1993; 4(4):230-3.

6. Symons AL, Townsend GC, Hughes TE. Dental characteristics of patients with Duchenne muscular dystrophy. ASDC J Dent Child 2002;69(3):234 277-83.

7. Symons AL, Stritzel F, Stamation J. Anomalies associated with hypodontia of the permanent lateral incisor and second premolar. J Clin Pediatr Dent 1993;17(2):109-11.

8. Symons AL, Taverne AA. A family case report: disturbances in tooth form and eruption of the second premolar. Aust Orthod J 1996;14(3):168-71.

9. Brown KS. Evolution and development of the dentition. Birth Defects Orig Artic Ser 1983;19(1):29-66.

10. Williams SA, Ogden AR. Failure of eruption associated with anomalies of the dentition in siblings. Pediatr Dent 1988; 10(2):130-6.

11. Mimault C, Giraud G, Courtois V, et al. Proteolipoprotein gene analysis in 82 patients with sporadic Pelizaeus-Merzbacher disease: duplications, the major cause of the disease, originate more frequently in male germ cells, but point mutations do not. The Clinical European Network on Brain Dysmyelinating Disease. Am J Hum Genet 1999;65(2):360-9. 